\title{
Admission LDL-C and long-term mortality in patients with acute aortic dissection: a survival analysis in China
}

\author{
Xin Zeng', Xuan Zhou' ${ }^{2}$, Xue-Rui Tan ${ }^{3}$, Ye-Qun Chen ${ }^{3}$ \\ ${ }^{1}$ Department of Geriatrics, The First Affiliated Hospital of Shantou University Medical College, Shantou, China; ${ }^{2}$ Department of Internal Medicine, \\ Fujian Medical University Xiamen Humanity Hospital, Xiamen, China; ${ }^{3}$ Department of Cardiovascular Internal Medicine, The First Affiliated \\ Hospital of Shantou University Medical College, Shantou, China \\ Contributions: (I) Conception and design: XR Tan, YQ Chen; (II) Administrative support: X Zeng; (III) Provision of study materials or patients: X \\ Zeng; (IV) Collection and assembly of data: X Zeng, X Zhou; (V) Data analysis and interpretation: X Zeng, X Zhou; (VI) Manuscript writing: All \\ authors; (VII) Final approval of manuscript: All authors. \\ Correspondence to: Xue-Rui Tan; Ye-Qun Chen. Department of Cardiovascular Internal Medicine, The First Affiliated Hospital of Shantou University \\ Medical College, 57 Changping Road, Jinping District, Shantou 515000, China. Email: tanxuerui@vip.sina.com; 13729294832@139.com.
}

\begin{abstract}
Background: The level of blood lipid is closely related to prognosis in cardiovascular diseases. This study aims to analyze the effect of serum low-density lipoprotein cholesterol (LDL-C) levels on the long-term mortality in acute aortic dissection (AAD). A lower admission LDL-C level is associated with an increased risk of long-term mortality in AAD.

Methods: We analyzed the data of 284 patients with AAD admitted to the First Affiliated Hospital of Shantou University Medical College from February 2016 to September 2019. Patients were followed up post-discharge. All patients were divided into either an LDL-C low-level group or an LDL-C high-level group according to the optimal cut-off point obtained by the receiver operating characteristic (ROC) curve. The endpoint outcome was long-term mortality in AAD. A survival analysis and Cox proportional hazards model were used.

Results: According to the Youden index, the optimal cut-off point for LDL-C was $2.755 \mathrm{mmol} / \mathrm{L}$. The Kaplan-Meier survival analysis curves showed that the long-term mortality of the LDL-C low-level group $(<2.755 \mathrm{mmol} / \mathrm{L})$ was significantly higher than that of the LDL-C high-level group $(\geq 2.755 \mathrm{mmol} / \mathrm{L})(\log -$ rank $\left.\chi^{2}=13.912, \mathrm{P}<0.001\right)$. After multivariate Cox regression analysis, $\mathrm{LDL}-\mathrm{C}<2.755 \mathrm{mmol} / \mathrm{L}$ was still significantly associated with long-term mortality in AAD (HR=3.287, 95\% CI: 1.637-6.600, P=0.001). In addition, cystatin $\mathrm{C}$ was also an independent risk factor for the long-term prognosis of AAD (HR=1.253, 95\% CI: $1.057-1.486, \mathrm{P}=0.009)$.
\end{abstract}

Conclusions: A lower admission LDL-C level may be associated with an increased risk of long-term mortality in AAD.

Keywords: Acute aortic dissection (AAD); low-density lipoprotein cholesterol (LDL-C); cholesterol paradox; long-term mortality rate

Submitted Jul 16, 2021. Accepted for publication Aug 20, 2021.

doi: 10.21037/atm-21-3511

View this article at: https://dx.doi.org/10.21037/atm-21-3511

\section{Introduction}

Acute aortic dissection (AAD) is a relatively rare cardiovascular disease, which has severe chest and back pain with a tearing quality as the primary clinical manifestation and a high mortality rate, especially in the case of delayed diagnosis and treatment (1). Epidemiological investigation shows that the mortality of acute Stanford type A aortic dissection (ATAAD) without surgical treatment increases by $1-2 \%$ per hour within 24 hours (2). Therefore, it is essential to quickly identify and diagnose high-risk patients by evaluating risk factors and conducting risk stratification. At present, it is recognized that the primary pathogenesis of aortic dissection is atherosclerosis 
and hypertension. As such, in 2014, the European Society of Cardiology's Guidelines for Diagnosis and Treatment of Aortic Diseases recommended that patients should monitor their blood pressure for an extended period after discharge and control it within an ideal range (3).

Dyslipidemia plays a key role in the occurrence and development of atherosclerosis and major cardiovascular events $(4,5)$. In these conditions, low-density lipoprotein (LDL) is transformed from very-low-density lipoprotein (VLDL) and high-density lipoprotein (HDL). As 50\% of LDL particles are cholesterol, this constitutes the principal cholesterol in blood lipoprotein. Many studies have suggested that an elevated serum low-density lipoprotein cholesterol (LDL-C) level is the main risk factor for atherosclerosis. The risk of atherosclerosis cardiovascular disease (ASCVD) is also significantly increased in patients with elevated serum LDL-C levels $(6,7)$. Mainstream opinions suggest that reducing the LDL-C level is the primary goal of blood lipid management in patients with cardiovascular diseases $(8,9)$. However, there is no clear guidance on whether it is necessary to delay the progression of atherosclerosis by lowering blood lipid levels to prevent the recurrence of aortic dissection or the risk of other cardiovascular diseases.

Several studies have shown that levels of total cholesterol (TC) and high-density lipoprotein cholesterol (HDL-C) are closely related to the in-hospital mortality of AAD patients (10-12). In a recent report explored the prediction of plasma biomarkers for the short-term prognosis of patients with $\mathrm{AAD}$, includes admission LDL-C level. The study shows that an independent association has been shown between LDL-C and in-hospital mortality in patients with AAD (OR 0.373, 95\% CI: 0.148-0.940) (13). However, such studies are limited to the short-term prognosis of patients. In addition, the relationship between LDL-C, which is closely related to atherosclerosis, and prognosis in AAD has not been established. Therefore, this study retrospectively analyzed the data of patients with $\mathrm{AAD}$ and the correlation between serum LDL-C levels and long-term mortality in AAD.

We present the following article in accordance with the STARD reporting checklist (available at https://dx.doi. org/10.21037/atm-21-3511).

\section{Methods}

\section{Participants}

Consecutive patients with suspected acute aortic dissection admitted to the emergency center of the First Affiliated
Hospital of Shantou University Medical College from February 2016 to September 2019 were eligible to be included in this study. The inclusion criterion was AAD diagnosed by multi-slice spiral CT scanning, using the diagnostic criteria specified in the 2014 European Society of Cardiology Guidelines for Diagnosis and Treatment of Aortic Diseases (3). The exclusion criteria were as follows: (I) iatrogenic aortic dissection, a history of cardiac or aortic surgery, traumatic aortic dissection, or AAD complicated with Marfan syndrome or vasculitis; (II) non-first diagnosis with aortic dissection; (III) in-hospital death; and (IV) incomplete data.

The study protocol was approved by the Medical Ethics Committee of The First Affiliated Hospital of Shantou University Medical College (no. 2020-141). All subjects signed informed consent, in line with the Declaration of Helsinki (as revised in 2013).

\section{Data collection and definitions}

The baseline characteristic data included age, sex, history of smoking, history of hypertension, history of diabetes mellitus, history of coronary heart disease, and admission systolic/diastolic blood pressure. Other recorded clinical characteristics included hematological indexes (white blood cell count, neutrophil ratio, hemoglobin concentration, LDL-C, serum creatinine, urea nitrogen, uric acid, estimated glomerular filtration rate, cystatin-C, and glycosylated hemoglobin), the in-hospital drug treatment plan, imaging examinations, and surgical interventions. A medical history of hypertension and diabetes was determined according to the previous medical history of antihypertensive and hypoglycemic drug use. The history of coronary heart disease was based on the previous medical history documented in the hospital medical records, or previous coronary angiography that showed at least one of the following had coronary stenosis $\geq 50 \%$ : the left main artery, left anterior descending branch, left circumflex branch, or right coronary artery. Admission systolic blood pressure/diastolic blood pressure was determined by taking the brachial artery blood pressure measured when the patient's left upper limb or right upper limb was at the same horizontal line with the heart, measuring the blood pressure of both left and right upper limbs, and recording the higher value. All blood indexes were detected within 48 hours of admission. The estimated glomerular filtration rate was converted by an MDRD simplified formula (14). The choice of surgical intervention was determined by experienced cardiac surgeons 


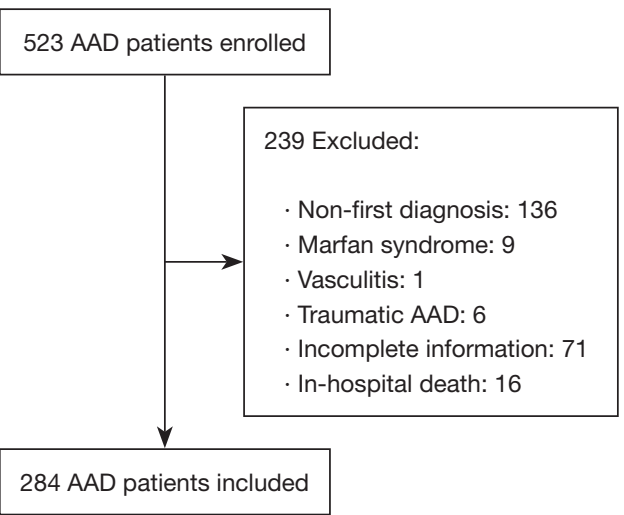

Figure 1 Patient selection. AAD, acute aortic dissection.

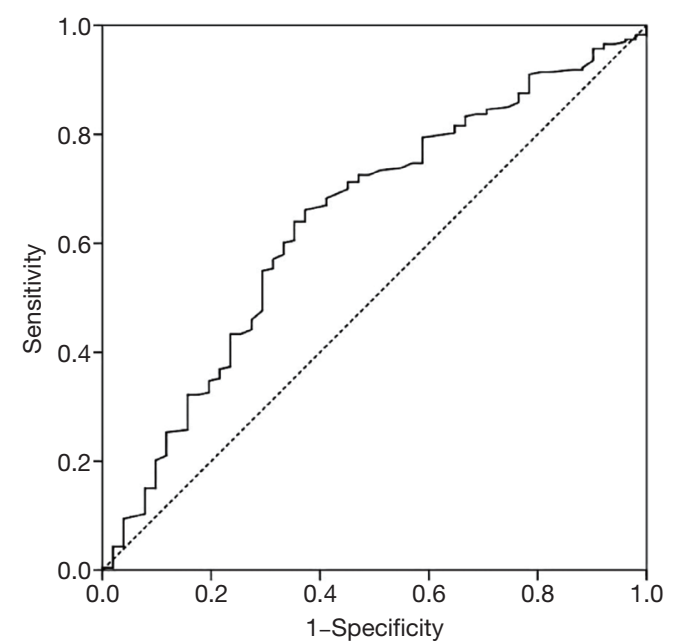

Figure 2 Receiver operating characteristic (ROC) curves for admission serum LDL-C to predict the long-term mortality risk of AAD. LDL-C, low-density lipoprotein cholesterol; AAD, acute aortic dissection.

or cardiovascular interventionists in the hospital.

\section{Outcome}

The endpoint outcome was the long-term mortality of patients with AAD admitted to hospital from February 2016 to April 2020. The main end event of this study was death caused by various reasons, including direct or recurrent $\mathrm{AAD}$ and was based on the patients' survival status at the follow-up deadline.

\section{Statistical analysis}

Continuous variables are presented as the mean \pm SD if normally distributed or as the median and interquartile range for non-normal distributions. Baseline characteristics were compared among the groups using ANOVA or Wilcoxon rank-sum tests, as appropriate. A receiver operating characteristic (ROC) analysis was performed to determine the optimal cut-off for admission serum LDL-C values in predicting the presence of events with high sensitivity and specificity. Kaplan-Meier survival curves stratified by admission serum LDL-C levels were constructed and compared using the log-rank test. A multivariate Cox proportional hazard model was used to estimate the role of admission serum LDL-C level as a factor affecting $\mathrm{AAD}$ mortality.

\section{Results}

\section{Patients enrolled and follow-up}

From February 2016 to September 2019, 523 patients were diagnosed as AAD in the First Affiliated Hospital of Shantou University Medical College. A final number of 284 AAD patients were included in this study after screening according to the exclusion criteria (non-first diagnosis, $\mathrm{n}=136$; Marfan syndrome, $\mathrm{n}=9$; vasculitis, $\mathrm{n}=1$; traumatic AAD, $\mathrm{n}=6$; incomplete data, $\mathrm{n}=71$; and in-hospital death, $\mathrm{n}=16$ ) (Figure 1). During the median follow-up period of 18 months (range, 9-27 months), 51 patients died, while 233 patients survived.

\section{The receiver operating characteristic (ROC) analysis}

A ROC analysis was performed to determine the optimal cut-off value for admission LDL-C in predicting the presence of adverse events with high sensitivity and specificity (Figure 2). According to the results, the AUC was 0.641 (95\% CI: 0.556-0.726, $\mathrm{P}=0.002$ ), and the Youden index was 0.288 . The optimal cut-off point was $2.755 \mathrm{mmol} / \mathrm{L}$ for admission LDL-C (sensitivity: $66.1 \%$, specificity: $62.7 \%)$. According to the optimal cut-off point, patients were divided into a low LDL-C level group $(<2.755 \mathrm{mmol} / \mathrm{L})$ and a high LDL-C level group $(\geq 2.755 \mathrm{mmol} / \mathrm{L})$.

\section{The baseline characteristics between the two groups}

Comparison of the baseline characteristics between the two groups showed no statistical differences in the majority of the basic demographic variables, as shown in Table 1. There were significant differences in admission SBP $(159.57 \pm 29.10$ 
Table 1 Baseline characteristics according to admission serum LDL-C levels in AAD patients

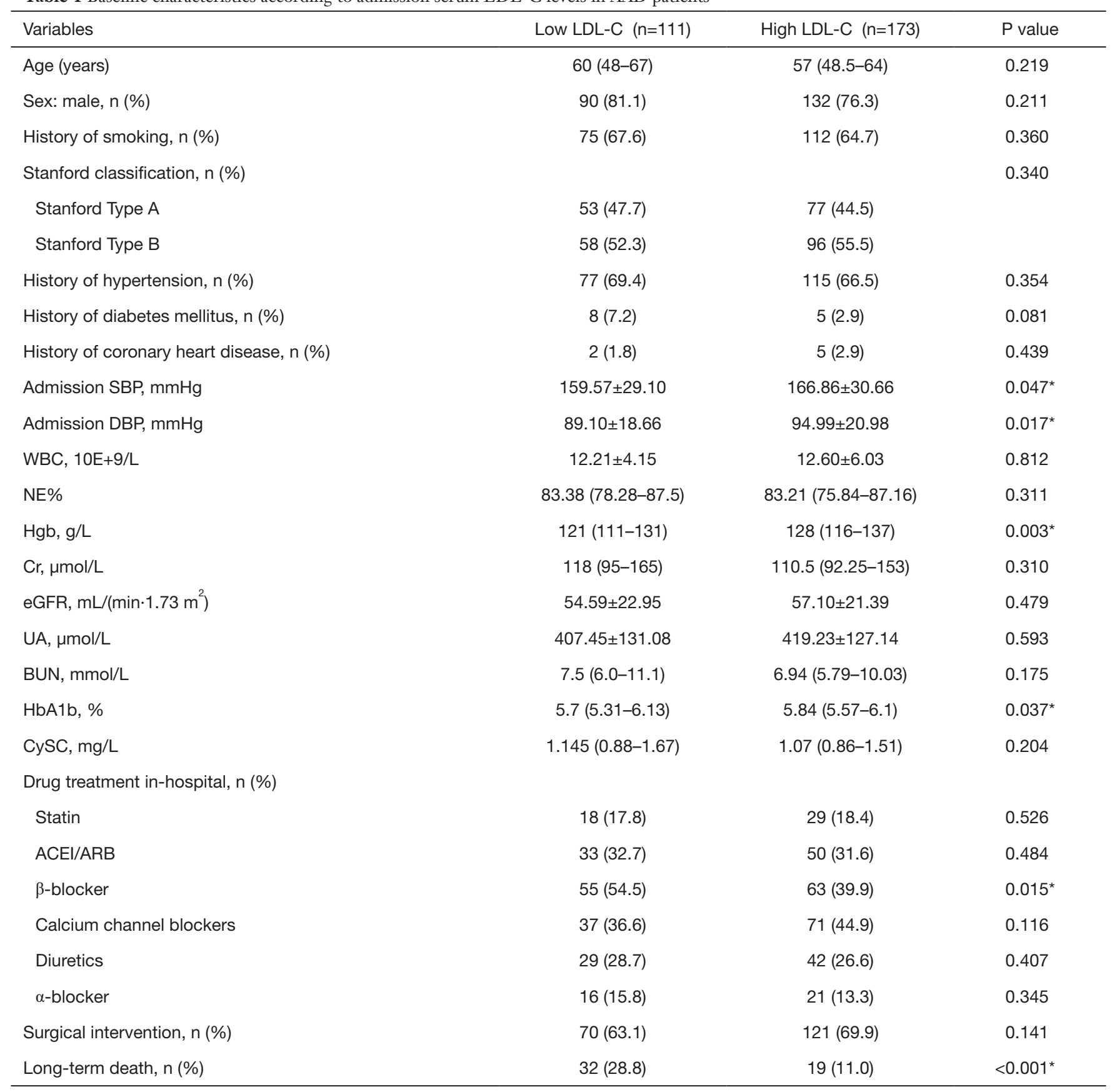

*, there were significant differences between the low LDL-C group and the high LDL-C group. AAD, acute aortic dissection; LDL-C, low-density lipoprotein cholesterol; SBP, systolic blood pressure; DBP, diastolic blood pressure; WBC, white blood cell count; NE, neutrophil ratio; Hgb, hemoglobin; Cr, creatinine; eGFR, estimated glomerular filtration rate; UA, uric acid; BUN, blood urea nitrogen; $\mathrm{HbA1b}$, glycosylated hemoglobin; CySC, cystatin-C.

vs. $166.86 \pm 30.66, \mathrm{P}=0.047), \mathrm{DBP}(89.10 \pm 18.66$ vs. 94.99 $\pm 20.98, \mathrm{P}=0.017), \mathrm{Hgb}[121(111-131)$ vs. 128 (116-137), $\mathrm{P}=0.003], \mathrm{HbA1c}$ [5.7 (5.31-6.13) vs. 5.84
(5.57-6.1), $\mathrm{P}=0.037]$, and $\beta$-blockers [55 (54.5\%) vs. 63 (39.9\%), $\mathrm{P}=0.015]$. In addition, the mortality rate of $\mathrm{AAD}$ patients in the low LDL-C group was significantly higher 


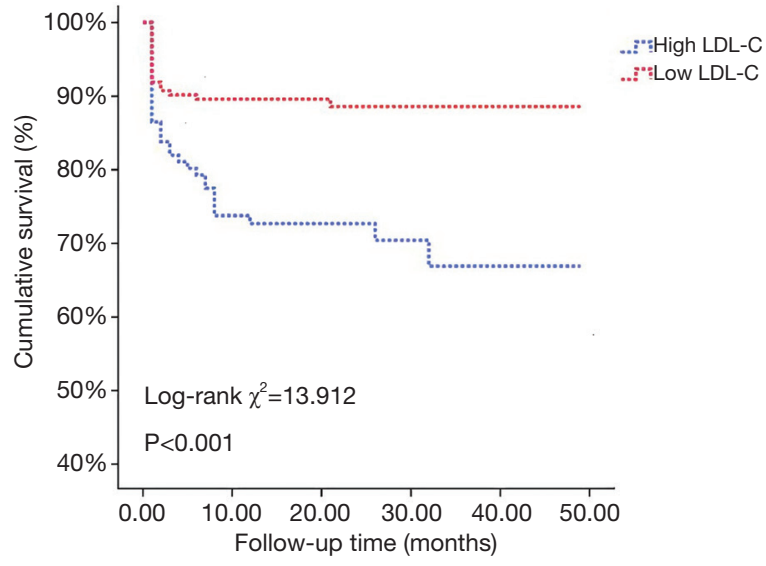

Figure 3 Kaplan-Meyer curves showing the cumulative long-term survival rate according to serum LDL-C level in patients with AAD. LDL-C, low-density lipoprotein cholesterol; AAD, acute aortic dissection.

than that in the high LDL-C group [32 (28.8\%) vs. 19 $(11 \%), \mathrm{P}<0.001]$.

\section{Kaplan-Meier survival analysis}

The Kaplan-Meier survival curves grouped by admission serum LDL-C levels are illustrated in Figure 3. The logrank test showed significant differences in the longterm cumulative survival rate among patients grouped by admission serum LDL-C level. The cumulative survival rate of the high LDL-C group was significantly higher than that of the low LDL-C group (log-rank $\left.\chi^{2}=13.912, \mathrm{P}<0.001\right)$.

\section{Univariate and multivariate Cox proportional hazards model}

A Cox regression model was used to identify the prognostic value of LDL-C levels for long-term mortality. Univariate and multivariate Cox regression analyses for long-term mortality in all the enrolled patients are presented in Tables 2 and 3, respectively. Since the mortality rate is higher in the low LDL-C group, the high LDL-C group is taken as the reference object. Compared to the high LDL-C level group, the risk of long-term all-cause mortality rate in the low LDL-C level group was significantly higher (HR $=2.732$, 95\% CI: $1.548-4.821, \mathrm{P}=0.001)$. Other indicators significantly related to the long-term mortality of AAD are shown in Table 2.

The univariate Cox regression analysis results were combined with the clinical variables to perform the multivariate Cox regression analysis, as shown in Table 3. After adjusting for age, sex, admission SBP, admission DBP, history of smoking, history of hypertension, history of diabetes mellitus, history of coronary heart disease, Stanford classification, Hgb, cystatin C (CySC), HbAlc, $\beta$-blocker, $\alpha$-blocker, and surgical intervention, the low LDL-C level group was significantly correlated with the longterm mortality of AAD (HR =3.287, 95\% CI: 1.637-6.600, $\mathrm{P}=0.001)$. Moreover, increased age ( $\mathrm{HR}=1.051,95 \% \mathrm{CI}$ : 1.020-1.083, $\mathrm{P}=0.001)$, Stanford type A (HR $=2.177,95 \%$ CI: $1.037-4.568, \mathrm{P}=0.040)$, and $\mathrm{CySC}(\mathrm{HR}=1.253,95 \%$ CI: $1.057-1.486, \mathrm{P}=0.009)$ were independent risk factors for $A A D$. Surgical intervention was a protective factor for the long-term survival of AAD (HR $=0.279$, 95\% CI: 0.135 $0.578, \mathrm{P}=0.001)$.

\section{Discussion}

In this study, we retrospectively analyzed the relationship between LDL-C levels and long-term all-cause mortality in 284 AAD patients. The Kaplan-Meier survival curves showed that the long-term mortality rate of the low LDL-C group was significantly higher than that of the high LDL-C group. After multivariate Cox regression analysis, a significant correlation remained between serum LDL-C levels and long-term death risk in $\operatorname{AAD}(\mathrm{P}<0.05)$. Thus, serum LDL-C can be used as an independent predictor of long-term mortality in $\mathrm{AAD}$. In contrast to previous studies, our study shows that a lower admission LDL-C level may be related to an increased risk of long-term mortality. Moreover, we found that increased age and Stanford type A were independent risk factors for all-cause mortality in $\mathrm{AAD}$, and surgical intervention was a protective factor for the long-term survival of AAD patients.

High cholesterol has always been considered as one of the main risk factors for cardiovascular diseases. Many studies have shown that elevated LDL-C levels can increase the risk of coronary heart disease, hypertension, and other diseases $(15,16)$. The 2018 American Heart Association (AHA) Guideline on the Management of Blood Cholesterol recommends that patients with ASCVD be treated with a high-intensity statin or the maximum tolerable dose of statin to reduce LDL-C levels. According to the guideline, the lower the LDL-C level, the greater the reduction in the prognostic risk for patients with ASCVD (17). Piao et al. (18) reported that statins could improve the prognosis of patients with acute myocardial infarction whose LDL-C baseline level was lower than $50 \mathrm{mg} / \mathrm{dL}$. However, a large- 
Table 2 Predictors of long-term all-cause mortality in patients with AAD by univariate Cox analysis

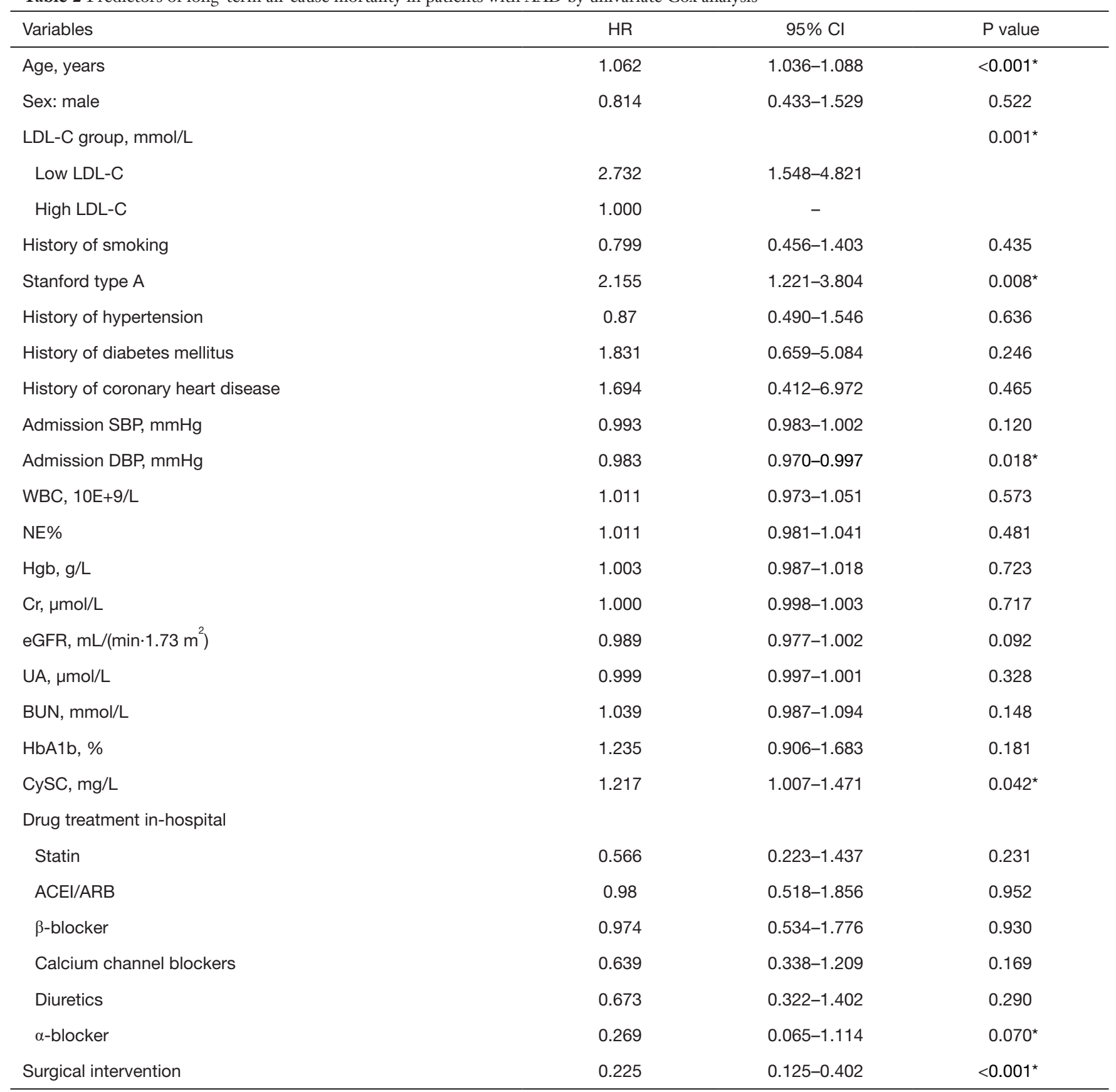

*, a significant difference related to the long-term mortality of AAD. HR, Hazard ratio; CI, Confidence Interval; AAD, acute aortic dissection; LDL-C, low-density lipoprotein cholesterol; SBP, systolic blood pressure; DBP, diastolic blood pressure; WBC, white blood cell count; $\mathrm{NE}$, neutrophil ratio; Hgb, hemoglobin; Cr, creatinine; eGFR, estimated glomerular filtration rate; UA, uric acid; BUN, blood urea nitrogen; $\mathrm{HbA1b}$, glycosylated hemoglobin; CySC, cystatin-C.

scale, prospective, multicenter study by Ahn et al. (19) in South Korea indicated that patients with acute myocardial infarction who received an LDL-C treatment target value $<70 \mathrm{mg} / \mathrm{dL}$ did not show better clinical results. In addition, many studies have shown that low cholesterol is related to a worse prognosis and higher mortality in cardiovascular diseases, which is called the "cholesterol paradox". Many studies on cardiovascular diseases support this conclusion. 
Table 3 Predictors of long-term all-cause mortality in patients with AAD by multivariate Cox analysis

\begin{tabular}{|c|c|c|c|}
\hline Variables & $\mathrm{HR}$ & $95 \% \mathrm{Cl}$ & $P$ value \\
\hline Sex: male & 0.829 & $0.285-2.413$ & 0.730 \\
\hline \multicolumn{4}{|l|}{ LDL-C group } \\
\hline Low LDL-C, mmol/L & 3.287 & $1.637-6.600$ & $0.001^{*}$ \\
\hline Admission SBP, mmHg & 1.006 & $0.990-1.023$ & 0.478 \\
\hline Admission DBP, mmHg & 0.989 & $0.960-1.018$ & 0.443 \\
\hline History of smoking & 1.039 & $0.389-2.776$ & 0.939 \\
\hline History of hypertension & 0.578 & $0.291-1.146$ & 0.116 \\
\hline Stanford type A & 2.177 & $1.037-4.568$ & $0.040^{*}$ \\
\hline $\mathrm{Hgb}, \mathrm{g} / \mathrm{L}$ & 1.009 & $0.989-1.028$ & 0.382 \\
\hline CySC, mg/L & 1.253 & $1.057-1.486$ & $0.009^{*}$ \\
\hline HbAlc, \% & 1.351 & $0.918-1.987$ & 0.127 \\
\hline$\beta$-blocker & 1.075 & $0.517-2.236$ & 0.846 \\
\hline$\alpha$-blocker & 0.294 & $0.065-1.321$ & 0.110 \\
\hline Surgical intervention & 0.279 & $0.135-0.578$ & $0.001^{*}$ \\
\hline
\end{tabular}

*, a significant difference related to the long-term mortality of AAD. HR, Hazard ratio; Cl, Confidence Interval; AAD, acute aortic dissection; LDL-C, low-density lipoprotein cholesterol; SBP, systolic blood pressure; DBP, diastolic blood pressure; Hgb, hemoglobin; HbA1b, glycosylated hemoglobin; CySC, cystatin-C.

For example, some studies on heart failure and acute myocardial infarction have shown that a lower baseline TC or LDL-C increases the risk of patient mortality (20-23).

The cholesterol paradox has also been reported in AAD. A study on TC and hospital mortality in AAD patients showed that the hospitalization all-cause mortality of patients in a low TC group was significantly higher than that of patients in a high TC group (15.4\% vs. 9.0\%, $\mathrm{P}<0.001)(10)$. These findings also verify the results of this study. Some studies have shown that serum HDL-C is negatively correlated with the hospital mortality of AAD patients (11). Other researchers have postulated that a lower HDL-C level may affect cell differentiation, proliferation, and extracellular matrix reconstruction, which may indicate the molecular mechanism of the phenomenon $(12,24)$. In such a reverse epidemiological phenomenon, a lower LDL-C level will also adversely affect the prognosis of patients, which is consistent with the findings of this study.
At present, the mechanism of this reverse epidemiology is unclear but may be related to the following factors:

One factor is immune inflammation. Some scholars have pointed out that the mechanism of the cholesterol paradox can be partly explained by immune inflammation (25). Adipose tissue can increase the expression of soluble tumor necrosis factor- $\alpha(\mathrm{TNF}-\alpha)$ receptors (sTNFR-I and sTNFRII) in the blood, which may reduce the expression of the related biological effects of TNF- $\alpha$, thus providing protection for obese patients (26). Higher circulating lipoprotein levels can also bind and degrade lipopolysaccharides responsible for the release of inflammatory factors. Some studies have shown that the level of hyperlipidemia can change the immune cells of the human body and induce chronic inflammation (27). However, the results from recent research are not consistent. For instance, some studies have shown that chronic inflammation can significantly reduce blood lipid levels; in one study the level of LDL-C was negatively correlated 
with female C-reactive protein (CRP) $(28,29)$. However, the research on this theoretical mechanism is still in progress.

Another factor is nutritional support. Although elevated LDL-C levels can lead to atherosclerosis and ASCVD, higher cholesterol levels may reflect the better nutritional status of patients with cardiovascular risk events. In particular, elderly coronary heart disease patients generally suffer from malnutrition, and failure to supplement reasonable nutrition and energy over time may lead to a higher risk of adverse events, such as multiple organ failure and increased mortality (30). When cardiovascular emergencies such as aortic dissection occur, a lower baseline cholesterol level may not be able to tolerate the excessive energy consumption associated with an acute medical stress reaction, resulting in intestinal edema that further affects food intake and intestinal absorption and reduces the quality of life (31). Therefore, good-or even excessivenutritional support may be an effective protective factor for the prognosis of patients with cardiovascular diseases.

This study shows that serum CySC is an independent risk factor for the long-term prognosis of $\mathrm{AAD}$. CySC is a protein encoded by the CST3 gene. Its molecular weight is light, it can filter freely through the glomerulus, the renal tubular epithelial cells do not secrete or absorb it, and the kidney is its only metabolic pathway. CySC is less affected by sex, age, weight, diet, liver function changes, and other drugs. Therefore, in recent years, many studies have indicated that $\mathrm{CySC}$ is superior to serum $\mathrm{Cr}$ and $\mathrm{BUN}$ in detecting renal function and that it is a new sensitive index for evaluating eGFR (32). Patients with AAD, especially those with abdominal aorta dissection, suffer from renal ischemia due to stenosis or obstruction of various renal artery branches, resulting in pre-renal acute kidney injury. In addition, patients with AAD generally need a CT angiography examination of the aorta, and the excretion of the contrast media into the injured kidney slows down, which can cause iatrogenic damage such as contrast media nephropathy, thus increasing the burden of renal function. Studies have shown that the expression of $\mathrm{CySC}$ in deceased $\mathrm{AAD}$ patients is significantly higher than that in surviving patients $(\mathrm{P}<0.01)$. When $\mathrm{CySC} \geq 1.1 \mathrm{mg} / \mathrm{L}$, it is considered to be a predictor of long-term mortality in ATAAD (HR $=1.49,95 \%$ CI: $1.10-7.36, \mathrm{P}=0.013$ ) (33). CySC may play an important role in the balance between cysteine protease and its inhibitors, thus promoting the remodeling of the blood vessel wall (34). In addition, a high CySC level is also associated with an increased risk of other cardiovascular diseases. Shlipak et al. (35) reported that a higher CySC level was associated with a higher risk of cardiovascular disease death. They concluded that CySC was a stronger predictor of the risk of cardiovascular events and death in elderly patients than serum Cr. The effect of CySC on the prognosis of $\mathrm{AAD}$ needs further study.

AAD is a critical cardiovascular disease that seriously threatens the life and health of Chinese. In recent years, with the improvement of medical staff's understanding of aortic disease and the improvement of imaging diagnosis, cardiovascular surgery, anesthesia and cardiopulmonary bypass technology, the diagnosis rate of AAD continues to improve, and the incidence of surgical death and complications is significantly reduced. However, as there is still a lack of large-scale prospective randomized controlled trial (RCT) studies on the treatment of AAD in the international community, there are still many disputes about the diagnosis and treatment of AAD in the industry. The high risk factors for AAD have been identified as follows: (I) various factors that increase aortic wall tension, such as hypertension; (II) Factors leading to structural abnormalities of the aortic wall, such as atherosclerosis, and hereditary connective tissue diseases (such as Marfan syndrome); (III) Other factors include pregnancy and iatrogenic AAD (3) . Therefore, regular monitoring, control of blood pressure and prevention of atherosclerosis are the key to prevent AAD. At the same time, good control of blood pressure is also an effective means to reduce the mortality of patients with AAD (36). However, more prospective studies are needed to determine the need for lipid management in patients with $\mathrm{AAD}$.

This study has several limitations. First of all, our study was a single-center study with a small sample size, which may have biased the analysis results. Secondly, we collected the serum LDL-C of patients at admission and during the in-hospital lipid-lowering treatment plan but did not track the blood lipid level and medication plan of patients after discharge. This may have led to the incorrect classification of research factors due to the change of blood lipid levels following discharge, making it impossible to analyze the influence of changes in serum LDL-C levels on long-term mortality. Thirdly, the serum LDL-C of the patients in this study was not collected when patients were fasting and may not have reflected the true blood lipid level of the patients.

\section{Conclusions}

Our study found that admission serum LDL-C might serve as a predictor of adverse long-term events in $\mathrm{AAD}$ patients. 
A lower admission LDL-C level may be associated with an increased risk of long-term mortality.

\section{Acknowledgments}

Funding: This work was supported by the Guangdong University Innovation Team Project (Nature) (no.2019KCXTD003) and Guangdong Medical Leading Talent, the First Affiliated Hospital, SUMC, China (2019-2022).

\section{Footnote}

Reporting Checklist: The authors have completed the STARD reporting checklist. Available at https://dx.doi. org/10.21037/atm-21-3511

Data Sharing Statement: Available at https://dx.doi. org/10.21037/atm-21-3511

Conflicts of Interest: All authors have completed the ICMJE uniform disclosure form (available at https://dx.doi. org/10.21037/atm-21-3511). The authors have no conflicts of interest to declare.

Ethical Statement: The authors are accountable for all aspects of the work in ensuring that questions related to the accuracy or integrity of any part of the work are appropriately investigated and resolved. The study protocol was approved by the Medical Ethics Committee of The First Affiliated Hospital of Shantou University Medical College (no. 2020141). All subjects signed informed consent, in line with the Declaration of Helsinki (as revised in 2013).

Open Access Statement: This is an Open Access article distributed in accordance with the Creative Commons Attribution-NonCommercial-NoDerivs 4.0 International License (CC BY-NC-ND 4.0), which permits the noncommercial replication and distribution of the article with the strict proviso that no changes or edits are made and the original work is properly cited (including links to both the formal publication through the relevant DOI and the license). See: https://creativecommons.org/licenses/by-nc-nd/4.0/.

\section{References}

1. Silaschi M, Byrne J, Wendler O. Aortic dissection: medical, interventional and surgical management. Heart
2017;103:78-87.

2. Sun LZ, Zhu ZM, Liu YM et al. Chinese expert consensus on diagnosis and treatment of aortic dissection. Chinese Journal of Thoracic Cardiovascular Surgery, 2017;33:641-54.

3. Erbel R, Aboyans V, Boileau C, et al. 2014 ESC Guidelines on the diagnosis and treatment of aortic diseases: Document covering acute and chronic aortic diseases of the thoracic and abdominal aorta of the adult. The Task Force for the Diagnosis and Treatment of Aortic Diseases of the European Society of Cardiology (ESC). Eur Heart J 2014;35:2873-926.

4. Hendrani AD, Adesiyun T, Quispe R, et al. Dyslipidemia management in primary prevention of cardiovascular disease: Current guidelines and strategies. World J Cardiol 2016;8:201-10.

5. Guan XM, Wu SL, Yang XL, et al. Association of total cholesterol, low-density lipoprotein cholesterol, and nonhigh-density lipoprotein cholesterol with atherosclerotic cardiovascular disease and cancer in a Chinese male population. Int J Cancer 2018;142:1209-17.

6. Nanna MG, Navar AM, Wojdyla D, et al. The Association Between Low-Density Lipoprotein Cholesterol and Incident Atherosclerotic Cardiovascular Disease in Older Adults: Results From the National Institutes of Health Pooled Cohorts. J Am Geriatr Soc 2019;67:2560-7.

7. Ji Y, Bai C. Research progress of hypertriglyceridemia and coronary heart disease. Heart Mind 2018;2:40-4

8. Bittner V. The New 2019 AHA/ACC Guideline on the Primary Prevention of Cardiovascular Disease. Circulation 2020;142:2402-4.

9. Guo YL, Chen JL. Enlightenment of European dyslipidemia management guidelines in 2019 on clinical practice in China. Chinese Circulation Journal 2020;35:105-8.

10. Liu X, Su X, Zeng H. Impact of admission serum total cholesterol level on in-hospital mortality in patients with acute aortic dissection. PakJ Med Sci 2016;32:939-43.

11. Liu XT, He XW, Tan R, et al. High-density lipoprotein cholesterol and in-hospital mortality in patients with acute aortic dissection. J Huazhong Univ Sci Technolog Med Sci 2016;36:364-7.

12. Li W, Guo T, Chai XP, et al. Study on the change of blood lipid level in patients with aortic dissection and its correlation with hospital death. Chinese Circulation Journal 2018;33:157-60.

13. Xie N, Zhang W, Li H, Zhou J,, et al. Admission Values of Plasma Biomarkers Predict the Short-Term Outcomes 
in Acute Aortic Dissection. Heart Surg Forum. 2021 Jan 19;24(1):E048-E054.

14. Kopple JD. National kidney foundation K/DOQI clinical practice guidelines for nutrition in chronic renal failure. Am J Kidney Dis 2001;37:S66-70.

15. Ueda P, Gulayin P, Danaei G. Long-term moderately elevated LDL-cholesterol and blood pressure and risk of coronary heart disease. PLoS One 2018;13:e0200017.

16. Borghi C, Cicero AF, Saragoni S, et al. Rate of control of LDL cholesterol and incident hypertension requiring antihypertensive treatment in hypercholesterolemic subjects in daily clinical practice. Ann Med 2014;46:97-102.

17. Grundy SM, Stone NJ, Bailey AL, et al. 2018 AHA/ ACC/AACVPR/AAPA/ABC/ACPM/ADA/AGS/APhA/ ASPC/NLA/PCNA Guideline on the Management of Blood Cholesterol: A Report of the American College of Cardiology/American Heart Association Task Force on Clinical Practice Guidelines. J Am Coll Cardiol 2019;73:e285-350.

18. Piao ZH, Jin L, Kim JH, et al. Benefits of Statin Therapy in Patients With Acute Myocardial Infarction With Serum Low-Density Lipoprotein Cholesterol $\leq 50 \mathrm{mg} / \mathrm{dl}$. Am J Cardiol 2017;120:174-80.

19. Ahn T, Suh SY, Lee K, et al. Clinical Outcomes according to the Achievement of Target Low Density LipoproteinCholesterol in Patients with Acute Myocardial Infarction. Korean Circ J 2017;47:31-5.

20. Charach G, Rabinovich A, Ori A, et al. Low levels of lowdensity lipoprotein cholesterol: a negative predictor of survival in elderly patients with advanced heart failure. Cardiology 2014;127:45-50.

21. Greene SJ, Vaduganathan M, Lupi L, et al. Prognostic significance of serum total cholesterol and triglyceride levels in patients hospitalized for heart failure with reduced ejection fraction (from the EVEREST Trial). Am J Cardiol 2013;111:574-81.

22. Cheng KH, Chu CS, Lin TH, et al. Lipid paradox in acute myocardial infarction-the association with 30-day inhospital mortality. Crit Care Med 2015;43:1255-64.

23. Reddy VS, Bui QT, Jacobs JR, et al. Relationship between serum low-density lipoprotein cholesterol and in-hospital mortality following acute myocardial infarction (the lipid paradox). Am J Cardiol 2015;115:557-62.

24. Torsney E, Pirianov G, Charolidi N, et al. Elevation of plasma high-density lipoproteins inhibits development of experimental abdominal aortic aneurysms. Arterioscler Thromb Vasc Biol 2012;32:2678-86.

25. Liu Y, Coresh J, Eustace JA, et al. Association between cholesterol level and mortality in dialysis patients: role of inflammation and malnutrition. JAMA 2004;291:451-9.

26. Mohamed-Ali V, Goodrick S, Bulmer K, et al. Production of soluble tumor necrosis factor receptors by human subcutaneous adipose tissue in vivo. Am J Physiol 1999;277:E971-5.

27. Zewinger S, Reiser J, Jankowski V, et al. Apolipoprotein C3 induces inflammation and organ damage by alternative inflammasome activation. Nat Immunol 2020;21:30-41.

28. Tang RK. Molecular mechanism of lipid metabolism disorder and liver and aorta damage in chronic inflammatory state. Chongqing: Chongqing Medical University, 2008.

29. Folsom AR, Pankow JS, Tracy RP, et al. Association of C-reactive protein with markers of prevalent atherosclerotic disease. Am J Cardiol 2001;88:112-7.

30. Liao D, Zhang XY, Tong XQ, et al. Application of subjective holistic evaluation in nutritional evaluation of elderly patients with cardiovascular and cerebrovascular diseases. Prevention and Treatment of Cardio-CerebralVascular Disease 2017;17:205-6.

31. Rahman A, Jafry S, Jeejeebhoy K, et al. Malnutrition and Cachexia in Heart Failure. JPEN J Parenter Enteral Nutr 2016;40:475-86.

32. Yang CH, Du RH. Research progress on clinical application of serum cystatin C. Hebei Medical Journal 2015;37:2355-6.

33. Feng WZ, Zhou JQ, Yu GM, et al. Association of serum cystatin $\mathrm{C}$ levels with mortality in patients with acute type A aortic dissection. Oncotarget 2017;8:101103-11.

34. Liu J, Sukhova GK, Sun JS, et al. Lysosomal cysteine proteases in atherosclerosis. Arterioscler Thromb Vasc Biol 2004;24:1359-66.

35. Shlipak MG, Sarnak MJ, Katz R, et al. Cystatin C and the risk of death and cardiovascular events among elderly persons. N Engl J Med 2005;352:2049-60.

36. Malek LA. Cardiac rehabilitation in patients with thoracic aortic disease: Review of the literature and design of a program. Heart Mind 2018;2:65-9

(English Language Editor: D. Fitzgerald)

Cite this article as: Zeng $\mathrm{X}$, Zhou $\mathrm{X}$, Tan $\mathrm{XR}$, Chen YQ. Admission LDL-C and long-term mortality in patients with acute aortic dissection: a survival analysis in China. Ann Transl Med 2021;9(16):1345. doi: 10.21037/atm-21-3511 